\title{
Was ist orthodoxe Kritische Theorie?
}

\author{
Weimar, 9.9.2016 \\ Fabian Freyenhagen (University of Essex)
}

Im Jahr 1919 stellte Lukács die Frage, „Was ist orthodoxer Marxismus?“. Bereits bei Lukács schwingt Ironie mit in dieser Frage - denn wenn wir mit Orthodoxie Strenggläubigkeit meinen, dann ist ein „mitleidiges Lächeln die angemessene Antwort“ (auf die Frage „Was ist orthodoxer Marxismus?“). Aber Lukács betont zugleich, dass wir die Frage nach Orthodoxie auch so verstehen und stellen können, dass sie eine andere Art der Antwort einlädt oder sogar erfordert. Wenn wir sie als eine Frage nach der Quintessenz verstehen, lautet Lukács' Antwort wie folgt: Die Quintessenz des Marxismus bestehe nicht in den Resultaten von Marx' Forschung oder eines „..., Glauben[s]' an diese oder jene These“ und auch nicht in der „Auslegung eines ,heiligen Buches”“. Stattdessen beziehe sich „Orthodoxie in Fragen des Marxismus ... vielmehr ausschließlich auf die Methode". ${ }^{1}$

In meinem Vortrag heute möchte ich nun Lukács' Frage im Bezug auf die Kritische Theorie neu stellen: „Was ist orthodoxe Kritische Theorie?“. Ich möchte Ihnen eine Herangehensweise ans Herz legen, nach der sich die Kritische Theorie in dreifacher Hinsicht als orthodox bezeichnen lässt.

Erstens, wenn wir Orthodoxie im Sinne der Quintessenz verstehen, dann lautet meine Frage, um es mit einem Titel eines bekannten Aufsatzes von Nancy Fraser zu sagen: „Was ist kritisch an der Kritischen Theorie?“. Denn das Kritische ist die Quintessenz der Kritischen Theorie, wie man bereits ihrem

\footnotetext{
${ }^{1}$ In Geschichte und Klassenbewußtsein, Berlin: Malik Verlag, 1923; reproduziert in Werke, Band 2 „Frühschriften II“, Neuwied: Herman Luchterhand Verlag, 1968; Hervorhebung im Original; hier S. 13.
} 
Namen entnehmen kann. Gemäß der heute vorherrschende Antwort auf die Quintessenz-Frage kann Kritische Theorie nur dann kritisch sein, wenn sie über ein Begründungsprogramm verfügt. Denn nur wenn die Kriterien der Kritik als allgemein teilbar ausgewiesen werden können, sei der Theoretiker berechtigt, diese in Anschlag zu bringen. Meine Position steht dieser Sichtweise diametral entgegen: Meiner Auffassung zufolge muss die Kritische Theorie, um kritisch zu sein, kein Begründungsprogramm umfassen - mehr noch, nur ohne ein solches Begründungsprogramm kann Kritische Theorie hinreichend kritisch sein.

Von Orthodoxie spreche ich auch deshalb, weil ich denke, dass wir Einsichten der ersten Generation der Frankfurter Schule wiederbeleben sollten, die in den gegenwärtig dominanten Strömungen entweder völlig vernachlässigt werden oder deren weitreichende Signifikanz übersehen wird. Ich werde daher, wenn ich folgenden für eine Neuorientierung der Kritischen Theorie plädiere, immer wieder auf Horkheimers Schriften der 30er Jahre zurückgreifen.

Drittens wird sich herausstellen, dass orthodoxe Kritische Theorie letztlich doch etwas mit Strenggläubigkeit zu tun hat - aller Ironie zum Trotz (und auch aller Sekularität).

Beginnen möchte ich mit zwei Elementen von Max Horkheimers wegweisendem Aufsatz „Traditionelle und kritische Theorie“ (1937). Dabei soll auch eine Problemstellung aufgezeigt werden, denn es gilt zunächst, verständlich zu machen, warum viele sich zu einem Begründungsprogramm hingezogen fühlen ja ein solches für die Kritische Theorie als notwendig erachten. 
Das erste Element, das ich aufgreifen möchte, ist Horkheimers

Charakterisierung dessen, was für eine Kritische Theorie ausgeschlossen ist: ausgeschlossen ist der unreflektierte Bezug auf die geltenden Kriterien der Verbesserung der Gesellschaft:

Die Kategorien des Besseren, Nützlichen, Zweckmäßigen, Produktiven, Wertvollen, wie sie in dieser Ordnung [d.i., der gegebenen gesellschaftlichen Struktur] gelten, sind [dem Kritischen Theoretiker] vielmehr sehr verdächtig und keineswegs außerwissenschaftliche Voraussetzungen, ... $(\$ 20)^{2}$

Die gesellschaftlich anerkannten Kriterien können also nicht einfach die normative Richtlinie vorgeben; sonst bleibt Kritik im Rahmen der existierenden Gesellschaft verhaftet, deren Missstände die Kritik ja erst auf den Plan gerufen hat. Gesellschaftlich anerkannte Kriterien einfach zu übernehmen erscheint insbesondere dann problematisch, wenn die bestehenden Missstände notwendig mit der gesellschaftlichen Ordnung verknüpft sind. In diesem Sinne könne eine Theorie, die kritisch ist, nicht produktiv, nützlich, oder konstruktiv sein (vgl. $\S 50)$.

Das zweite Element, das ich von Horkheimer aufgreifen will, dreht sich um die geschichtliche Situierung der Kritischen Theorie. Eine der wichtigsten Anliegen von Horkeimers Aufsatz besteht in der Historisierung von Kants Erkenntnistheorie. Er denkt Erkenntnisobjekt und Erkenntnissubjekt nicht als transzendental präformiert, sondern als sozial-geschichtlich:

\footnotetext{
2 Ich zitiere hier einfach durch Nennung der Absätze. [in Gesammelte Schriften 4: 180-1]
} 
Die Tatsachen, welche die Sinne uns zuführen, sind in doppelter Weise gesellschaftlich präformiert: durch den geschichtlichen Charakter des wahrgenommen Gegenstands und den geschichtlichen Charakter des wahrnehmenden Organs. $(\$ 14)^{3}$

Hieraus ergeben sich verschiedene Konsequenzen für die Kritische Theorie, von denen ich eine kurz herausgreifen möchte. Horkheimer schreibt: „Niemand kann sich zu einem anderen Subjekt machen als zu dem des geschichtlichen Augenblicks“; und er wendet sich „gegen die Annahme eines absoluten, übergeschichtlichen Subjekts oder gegen die Auswechselbarkeit der Subjekte, als ob man sich aus dem gegenwärtigen Augenblick hinaus und ganz im Ernst in jeden beliebigen hineinversetzen könnte“ (§48). ${ }^{4}$

Zusammengenommen mögen die beiden von mir umrissenen Elemente zu dem Gedanken verleiten, dass kritische Theorie eigentlich unmöglich ist - und zwar dem eigenen Verständnis nach. Denn wie kann eine Theorie kritisch sein, wenn sie weder auf die gesellschaftlich anerkannten Kriterien der Verbesserung zurückgreifen kann noch auf übergeschichtliche Subjekte oder Reaktionsweisen ${ }^{5}$

Der gegenwärtig dominanten Auffassung der Geschichte der Frankfurter Schule zufolge, setzt genau wegen dieser Problemstellung ein Lernprozess in dieser Theorietradition ein. Eine zweite, von Jürgen Habermas angeführte

\footnotetext{
${ }^{3}$ [GS, 4: 171.] 'Beide sind nicht nur natürlich, sondern durch menschliche Aktivität geformt; das Individuum jedoch erfährt sich selbst bei der Wahrnehmung als aufnehmend und passiv.' Siehe auch Nachtrag, §1.

${ }^{4}$ [GS, 4: 213-4.] In dem im darauffolgendem Heft erschienenen „Nachtrag“ zu „Traditionelle und kritische Theorie" spitzt Horkheimer den Punkt noch weiter zu, wenn er schreibt, dass die ,klassenmäßige Form' jeder Tätigkeit, allen menschlichen Reaktionsweisen, auch der Theorie, ihren Stempel aufprägt' (Nachtrag, §2).

${ }^{5}$ Insbesondere, von welchem Standpunkt spricht sie wenn sie die gegebene Welt gleichzeitig als ,schlechte Faktizität' und ,unwahres Ganzes' versteht (um respektive mit Marcuse in 1937 und Adorno der '40er Jahre zu sprechen)?
} 
Generation erkannte die angedeutete Problemstellung und blies zum Rückzug aus der Sackgasse:

Wer an einem Ort, den die Philosophie einst mit Ihren Letztbegründungen besetzt hielt, in einer Paradoxie verharrt, nimmt nicht nur eine unbequeme Stellung ein; er kann die Stellung nur halten, wenn mindestens plausibel zu machen ist, daß es keinen Ausweg gibt. Auch der Rückzug aus einer aporetischen Situation muß verlegt sein, sonst gibt es einen Weg, eben den zurück. Dies, meine ich, ist aber der Fall. ${ }^{6}$

Seither gilt es als ausgemacht, dass ein Begründungsprogramm für die Kritische Theorie wesentlich ist.

Diese Programm wurde dann unterschiedlich ausgeführt, und hier gibt es kleine aber feine Unterschiede - die einen orientieren sich im Vollzug der Habermas'schen Kehrtwende an Kant; die anderen an Hegel. Trotz aller Verschiedenheit gibt es dabei eine Konstante: die jeweiligen Begründungsprogramme sollen den Nachweis der moralischen Richtigkeit der kritischen Maßstäbe leisten. Wie dies zu bewerkstelligen ist, bleibt umstritten. Manche versuchen es durch Rückgriff auf etwas, was nicht direkt moralisch ist (wie die Voraussetzungen des kommunikativen Handelns); andere mit Hilfe einer Geschichtsphilosophie, die uns in die Lage versetzen soll, von historischen Lernprozessen zu sprechen; und wieder andere mit Bezug auf eine direkt moralische Grundlage, etwa das basale Recht auf Rechtfertigung.

Mein Ansatz unterscheidet sich von den soeben skizzierten dadurch, dass ich eine zweite Kehrtwende vorschlagen möchte, die jedoch nicht in der Wiederherstellung jeder These oder Behauptung der ersten Generation der Frankfurt Schule besteht (das wäre, mit Lukács gesprochen, orthodoxe Theorie

${ }^{6}$ J. Habermas, Der philosophische Diskurs der Moderne, S. 155; Hervorhebung im Original. 
im falschen Sinne - in dem Sinne, wie wir sie nur mitleidig belächeln können). Es geht darum, die „unbequeme Stellung“ in der Adorno und Horkheimer kritische Theorie situieren, als „haltbar“ - vor allem, philosophisch haltbar - zu verteidigen. Erlauben Sie mir, etwas von dieser Verteidigung kurz zu skizzieren und anzudeuten, wie wir in der kritischen Theorie weiter vorgehen können.

Mein erster Schritt besteht darin wieder ernst zu nehmen, dass Kritische Theorie - wie es 1937 bei Horkheimer heißt - mit der „Not der Gegenwart“ (§29) beginnt. Auch diese wird nicht einfach unreflektiert aufgenommen, aber der Schwerpunkt liegt erst einmal auf den Negativerfahrungen der Menschen entlang verschiedener Achsen (nicht allein der Ungerechtigkeit). Elend, Unfreiheit und Unrecht verlangen nach ihrer Abschaffung ${ }^{7}$ - und dazu versucht die Kritische Theorie beizutragen. Kritische Theoretiker sind Partisanen im Kampf um die Aufhebung des gesellschaftlichen Unrechts, der versklavenden Verhältnisse, in denen das Elend vorherrscht - sie haben keine Ambitionen neutrale Wissenschaftler zu sein, denen es um eine bloße Registrierung und Systematisierung von Tatsachen geht, ${ }^{8}$ und die Denken und Handeln (vor allem wissenschaftliches Denken und politisches Handeln) kategorisch trennen. Sie meinen auch nicht, dass wir einfach unter Absehung von den bestehenden Verhältnissen normative Theorien konstruieren können, die dann mit Hilfe der Sozialwissenschaften nur noch auf diese Verhältnisse angewendet werden müssten. Nein, es geht darum, eine parteiische Welt kritisch zu durchleuchten, geleitet von einem Interesse an der Emanzipation des Menschen aus versklavenden Verhältnissen. In einer solchen parteiischen Welt wird diese

7 Vgl. ND, in GS 6:203: "Weh spricht: vergeh”.

8 Vgl. Marcuse, 111. 
Parteinahme dann „parteiisch und ungerecht“ wirken (§31), was wiederum dazu führt, dass eine unabhängige, universelle Begründung notwendig erscheint. Dies ist aber, so lautet der erste Schritt meiner Antwort, voreilig. Denn wer so argumentiert, setzt voraus, dass es einen neutralen Ort oder eine neutrale Art und Weise der Begründung geben kann. Nun ist es aber gerade diese Voraussetzung, die ich mit Horkheimer bezweifeln will:

Es gibt keine Theorie der Gesellschaft, auch nicht die des generalisierenden Soziologen, die nicht politische Interessen mit einschlösse, über deren Wahrheit anstatt in scheinbar neutraler Reflexion nicht selbst wieder handelnd und denkend, eben in konkreter geschichtlicher Aktivität, entschieden werden müßte. (§35 [GS, 4:196]; vgl.

„Materialismus und Moral, 149)

Ich möchte Horkheimer darin folgen, dass es keinen neutralen Standpunkt gibt und auch nicht geben kann - und insofern als Begründungsprogramme einen solchen Standpunkt voraussetzen, sind sie damit disqualifiziert. Keine Theorie kann wirklich neutral sein. Jeglicher Versuch, neutral der parteiischen Welt gegenüber zu sein, kommt letztlich dem Status quo zugute. Gewollt oder ungewollt sind solche Versuche letztlich von den herrschenden Interessen geleitet.

Horkheimer bringt noch eine andere Überlegung ins Spiel, die wesentlich für meine Argumentation ist. Direkt anschließend an das obige Zitat, fährt er fort:

Daß der Intellektuelle sich so hinstellt, als bedürfe es zunächst einer von Ihm zu leistenden schwierigen Denkarbeit, um zwischen revolutionären, liberalistischen und faschistischen Zielen und Wegen die Wahl zu treffen, ist überhaupt verwirrend. [...] Die Avantgarde bedarf der Klugheit im politischen Kampf, nicht der akademischen Belehrung über ihren sogenannten Standpunt. (§35 [GS, 4: 196]) 
Mein zweiter Schritt besteht also in der These, dass zumindest bestimmte Werturteile nicht der Denkarbeit der Intellektuellen bedürfen (nicht der Theorie) - über manche Übel ist akademische Belehrung unnötig und sogar fehl am Platze.

Eine ähnliche Sichtweise findet sich auch bei Adorno in einem Vortrag von 1962 in der folgende Nebenbemerkung über ,Unfreiheit und Unterdrückung': diese seien „... das Übel, das so wenig eines philosophischen Beweises bedarf, daß es das Übel sei, wie daß es existiert, ....9 Vier Jahre später, in Negative Dialektik, geht Adorno dann aber noch einen Schritt weiter, wenn er schreibt, dass der neue kategorische Imperativ - d.i., der Imperativ, unser Denken und Handeln so einzurichten, dass Auschwitz nicht sich wiederhole, nicht Ähnliches geschehe - „so widerspenstig gegen seine Begründung [ist] wie einst die Gegebenheit des Kantischen“, mehr noch , „[i]hn diskursiv zu behandeln, wäre Frevel: ..." (GS, 6:358).

Ich sehe hierin eine Kritik am Begründungsprogramm, die ich teile. ${ }^{10}$ Die von der Not der Gegenwart ausgehende, Interessen-geleitete Kritische Theorie hat nicht die Aufgabe, so Horkheimer und Adorno, die Übel als solche zu begründen und das Interesse an Emanzipation diskursiv abzuleiten. Nein, wenn Adorno Glauben zu schenken ist, dann wäre dies nicht nur ein unnötiges, sondern ein tiefproblematisches Unterfangen („ein Frevel“).

Wie können wir diese These verstehen und auch nur damit beginnen, sie zu verteidigen? Als erstes möchte ich nochmals die Idee aufgreifen, dass wir historisch situiert sind und nicht die Position eines übergeschichtlichen Subjekts

9 „Wozu noch Philosophie“, in GS, 10.2: 465.

10 Siehe auch vgl. „Materialismus und Moral“, S. 133-4, 146 
einnehmen können. Wenn das wahr ist, schließt es gewisse

Begründungsstrategien aus. Zum Beispiel hat diese Überlegung negative

Konsequenzen für Honneths geschichtsphilosophische Begründungsstrategie. In Recht auf Freiheit besteht Honneth gegen Walzer und anderen darauf, dass eine Kritische Theorie nicht nur in der gesellschaftlichen Wirklichkeit verankert sein müsse, sondern es auch eines Nachweises der moralischen Vernünftigkeit Ihrer Standards bedürfe (S. 16). Gleichzeitig kritisiert er Forst (und andere) - zurecht, wie ich meine - dafür, dass eine Rechtfertigung unabhängig von sozialen und historischen Verhältnissen ,vollkommen leer' sei (S. 39n6). Stattdessen will Honneth auf eine post-metaphysische Version der Kantschen und Hegelschen Geschichtsphilosophie zurückgreifen. Ein zentrales Problem hierbei ist es, dass die Kriterien für den angeblich stattgefundenen historischen Lernprozess entweder selber historisch sind (und als solche nicht geeignet, die historischen Umstände und Wertvorstellungen unabhängig von diesen zu begründen) oder eben - wie Honneth bei Forst bemängelt - „vollkommen leer“. Deswegen ist auch nicht klar, warum Honneth in anderen Werken meint die Auffassung vertreten zu können, „soziale Inklusion“ und „Individualisierung“ taugten als Kriterien des Fortschritts. Denn das Dilemma, das ich gerade skizziert habe, besteht für das „Recht der Rechtfertigung“ ebenso wie für die Kriterien der „Sozialen Inklusion“ und „Individualisierung“.

Dies bringt mich zu einer weiteren Überlegung, die mir ebenso wichtig erscheint. Nicht nur ist Neutralität unmöglich, sondern „[e]s gehört ein bestimmtes Interesse dazu“, ausschlaggebende „Tendenzen zu erfahren und 
wahrzunehmen“ (§26 [GS, 4: 187]; siehe auch §35).11 Anders gesprochen: wir haben nur dann Zugang zur Wahrheit, wenn wir von einem bestimmte Interessen geleitet sind - dem „Interesse an der Aufhebung des gesellschaftlichen Unrechts“ (§50 [GS, 4: 216]). Der Zugang ist nicht unparteilich oder interessenneutral, sondern erfolgt durch ein Subjekt mit diesem Interesse nur so können wir die Wahrheit als solche wahr-nehmen. Hieraus folgt, dass es keine Begründung des die kritische Theorie leitende Interesses geben kann, denn jeder Zugang zur Wahrheit (und parallel, jeder Zugang zu Kriterien der Richtigkeit und zu Begründungsstrategien) setzt schon jenes Interesses voraus. Vielleicht ist so auch der „Frevel“ zu verstehen, von dem Adorno spricht: zu meinen, dass das emanzipatorische Interesse begründet werden muss, würde bedeuten, dass es noch etwas Wichtigeres gibt, was diese Begründung zuallererst liefert. Unrecht, Unfreiheit und Unterdrückung würden metaethisch nicht als für sich sprechend betrachtet. Und das bedeutete, dass ihre Abschaffung nicht als das Wichtigste gilt. Mehr noch, es bedeutete, dass Unrecht, Unfreiheit und Unterdrückung - zumindest im Prinzip - als gerechtfertigt gelten könnten, nämlich dann, wenn das Wichtigere, was sie begründen soll, auf eine bestimmte Weise beschaffen wäre. (Darauf zu antworten, dass das Wichtigere nicht so beschaffen sei oder sogar nicht so beschaffen sein könnte, wird der Problemlage nicht gerecht - denn entweder ist dann die Begründung (doch) überflüssig oder es wird übersehen, dass schon das Offenlassen der Möglichkeit, dass Unfreiheit und Unterdrückung keine Übel seien, problematisch ist - so ähnlich wie alle Beteuerungen der Verteidiger des Utilitarismus, dass es in der Wirklichkeit unter seiner Vorherrschaft nie zum Hängen Unschuldiger käme,

\footnotetext{
11 vgl. „Materialismus und Moral, 131.
} 
nicht ausreicht, um ihn zu verteidigen. Man kann auch falsch liegen, wenn man falsche Gründe für etwas an sich Richtiges anführt.)

Hier sollte kurz angemerkt werden, dass eine Ablehnung des Begründungsprogramms nicht die Ablehnung von Argumentation oder Philosophie oder Vernunft einschließt. Stattdessen bedeutet es nur eine andere Art in der Philosophie vorzugehen, eine andere Art der Argumentation, ein anderes Vernunftverständnis. Ganz besonders wesentlich in diesem Ansatz sind Strategien der Sichtbarmachung. Manche der Übel werden leicht einsichtig sein, aber insbesondere angesichts der Präformierung der Wahrnehmungsorgane und -gegenstände ist es nötig, zur Erfahrbarkeit und Sichtbarkeit der komplexeren Missstände durch Theorie beizutragen - sie uns zu erschließen. Ein wichtiger Aspekt dabei ist die Bekämpfung derer theoretischen Gebilde die - ob von ihren Vertretern gewollt oder ungewollt - die Sichtbarkeit der gesellschaftlichen Missstände verschleiern oder verdecken. Was die Kritische Theorie dabei als Übel sichtbar macht, muss dann nicht als solches begründet werden.

Angesichts der Kritik am Begründungsprogramm ist die Rede von Orthodoxie - von Strenggläubigkeit - doch nicht ganz fehl am Platz, trotz aller Ironie, mit der sie von Lukács (und mir) eingeführt wurde. Und auch bezüglich dieses Punktes, meine ich mich in Übereinkunft mit Horkheimer zu befinden, der ausführt:

Allgemeine Kriterien für die kritische Theorie als Ganzes gibt es nicht; ... Die kritische Theorie hat bei aller Einsichtigkeit einzelner Schritte und der Übereinstimmung ihrer Elemente mit den fortgeschrittensten traditionellen Theorien keine spezifische Instanz für sich als das mit ihr selbst verknüpfte Interesse an der Aufhebung des gesellschaftlichen Unrechts. (§50 [GS, 4: 216]) 
Hier wäre in einer Hinsicht ein guter Schlusspunkt erreicht, aber in anderer Hinsicht ist dem nicht so, und wir stehen eigentlich erst am Anfang. Lassen Sie mich mit zwei Bemerkungen schließen:

Zum einen möchte ich kurz auf einen Einwand eingehen, der Ihnen sicher schon auf den Lippen liegt: Wie kann die Kritische Theorie, wie ich sie skizziert habe, dem Vorwurf des Dogmatismus' und Irrationalismus' entgehen? Hat nicht Habermas doch recht, dass es sich hierbei um eine unbequeme Stellung, ja sogar eine aporetische handele, aus der wir besser täten, den Rückzug anzutreten?

Ich gebe gerne zu, dass mein Ansatz, den ich heute umrissen habe, in einer Hinsicht unphilosophisch und problematisch klingt: wenn Philosophie so verstanden wird, dass es in ihr um Begründungsprogramme gehe und um universelle Wahrheiten, dann ist die Traditionslinie der kritischen Theorie, für die ich heute Partei ergriffen habe, unphilosophisch oder sogar antiphilosophisch. Wenn man zudem der Überzeugung ist, dass ein Begründungsprogramm sowohl möglich als auch notwendig ist, und universelle Wahrheiten sowohl existieren als uns zugänglich sind, dann muss mein Ansatz unweigerlich als problematisch erscheinen.

Hierauf möchte ich wie folgt antworten: Wenn unsere Situation eine ist, in der Begründungsprogramme und das Festhalten an universeller Geltung fehlgeleitete Ambitionen sind - und was ich heute gesagt habe, hat dieses überaus komplexe Thema natürlich nicht im Geringsten abschließend behandeln können - aber wenn sie fehlgeleitete Ambitionen sind, dann ist es reflektierter und philosophischer, sich das bewusst zu machen und andere Strategien zu wählen, um dem Irrationalismus und Dogmatismus entgegenzuarbeiten: 
Strategien der Selbstreflexion, Distanzierung (u.a. durch genealogische Studien) und sogar Selbstironie, der Einbeziehung von und Bezugnahme auf die Stimmen, die ausgeschlossen wurden und werden - wie z.B. in den Arbeiten von Nancy Fraser, Amy Allen und anderen. Es ginge dann darum, es der Gesellschaft und sich selber unbequem zu machen - auch das gehört zur Quintessenz der Kritischen Theorie.

Solche Strategie mögen auch denjenigen offenstehen, die sich Begründungsprogrammen verschreiben, aber ist es eine Illusion zu glauben, dass sie (durch das Begründungsprogramm) mehr anbieten können. Im Gegenteil: es besteht die Gefahr, dass durch den Fokus auf das Begründungsprogramm die Strategien der Selbstreflexion und Selbstdistanzierung vernachlässigt oder sogar verzerrt werden. Dies kann geschehen, wenn alles in ein abstraktes Schema eingepresst wird oder ein spezifisches Begründungsprogramm den Blick verengt. Nehmen Sie beispielsweise Honneths Ansatz in Das Recht auf Freiheit: Er beschränkt sich in diesem Werk (und nicht zufälligerweise) darauf, die für die Reproduktion der gegenwärtigen Gesellschaftsordnung unverzichtbaren Praktiken normativ zu rekonstruieren. ${ }^{12}$ Verloren geht dabei die normative Rekonstruktion der subversiven Praktiken, die die herrschenden Werte und Institutionen destabilisieren könnten und die damit für unserer Selbstreflexion und die Emanzipation vielleicht von viel größerer Bedeutung sind.

Ich gebe gerne zu, dass Selbstreflexionsstrategien keine Garantien bieten - aber wenn das Begründungsprogramm wirklich fehlgeleitet ist, dann kann nichts und niemand Garantien anbieten; die ,unbequeme Stellung' (von der

\footnotetext{
12 Vgl. J. Schaub, 'Misdevelopments, Pathologies, and Normative Revolutions: Normative Reconstruction as Method of Critical Theory', Critical Horizons 16.2 (May 2015): 107-130.
} 
Habermas im Bezug auf Adorno und Horkheimer spricht) erweist sich dann in der Tat als ausweglos. Eine kritische Sozialphilosophie auszuarbeiten, die weder im schlechten Sinne dogmatisch ist noch sich eines Begründungsprogramms verschreibt, ist eine der wichtigsten philosophischen Aufgaben - und eine, der wir uns widmen sollten.

Sozialphilosophie beschränkt sich natürlich nicht auf kritische Selbstreflexion (und die darin eingeschlossene Kritik des Begründungprogramms). Ich habe mich heute lediglich darauf konzentriert, um zu umreißen, wie ich denke, dass die Tradition der Frankfurter Schule weiterzuführen ist.

Bereits bei Horkheimer gibt Auskunft über weitere Rollen der kritischen Sozialphilosophie, etwa die der Kritik der Sozialwissenschaften, die ich allesamt für bedeutsam halte. So führt er (im ,Nachtrag') aus:

Im Unterschied zum Betrieb der modernen Fachwissenschaft ist jedoch die kritische Theorie der Gesellschaft auch als Kritik der Ökonomie philosophisch geblieben: ihren Inhalt bildet der Umschlag der die Wirtschaft durchherrschenden Begriffe in Ihr Gegenteil, des gerechten Tauschs in die Vertiefung der sozialen Ungerechtigkeit, der freien Wirtschaft in die Herrschaft des Monopols, der Erhaltung des Lebens der Gesellschaft in die Verelendung der Völker. (N, §5 [GS, 4: 220])

Implizit wird hier Philosophie mit einer bestimmten Art der Begriffsarbeit gleichgesetzt - mit der kritischen Analyse ökonomischer (oder, weiter gefasst, gesellschaftlicher) Prozesse, die in Ihr Gegenteil umschlagen. Philosophisch ist eine solche Analyse, als diese Prozesse von Begriffen bestimmt werden und die 
Arbeit am Begriff - etwa die Rekonstruktion und das Kritisieren von

begrifflichen Permutationen - das Metier der Philosophie ist. ${ }^{13}$

Die von Honneth angeregte Konzeptualisierung gesellschaftlicher

Fehlentwicklungen als „sozialer Pathologien“ stellt ein gutes Beispiel einer solchen kritischen Begriffsarbeit dar. Nur sollten wir dieses

Forschungsprogramm zur sozialen Pathologie in einer veränderten Form fortführen, d.h. in einer Form, die mit meiner vorgeschlagenen zweiten

Kehrtwende vereinbar ist. Solch ein Forschungsprogramm sollte, erstens, seinen Ausgangspunkt von der herrschenden Not in der jetzigen Gesellschaft nehmen und den Mechanismen die sie verschleiern.

Zweitens bestünde eine Aufgabe der Kritischen Theorie dann darin zu klären, inwieweit diese Not mit der gesellschaftlich Struktur zusammenhängt und zu zeigen, dass krank sein in gewisser Weise normal ist angesichts der Umstände in denen wir leben (MM, §36). Dabei darf nicht von vorne herein ausgeschlossen werden (besonders nicht durch methodische Weichenstellungen), dass es nötig sein könnte, die gesellschaftliche Struktur und sogar unsere Vorstellung von sozialer Freiheit zu überwinden. Dies nicht auszuschließen führt zu keinem aktionistischen Revolutionismus von der Art wie ihn Adorno abgelehnt hat, sondern impliziert ein Festhalten daran, dass es möglich sein kann, dass eine Transformation des gesellschaftlichen Ganzen

\footnotetext{
${ }^{13}$ Es sollte unnötig sein klarzustellen, dass es sich hier nicht um Begriffsarbeit im Sinne der konzeptuellen Analyse der Angelsächsischen Philosophie handelt. Und auch nicht um Idealismus. Vielmehr geht es um die These, dass gesellschaftliche Prozesse nicht einfach von bestimmten Kernbegriffen, die sie kennzeichnen, zu trennen sind. Diese Begriffe werden nicht einfach vom Denken den Praktiken zugeschrieben, sondern im gewisse Sinn durch diese Prozesse „erzeugt“ - sie sind „Realabstraktionen“ (um mit Sohn-Rethal zu sprechen). Das Umschlagen von Begriffen ist somit nicht dem Umschlagen der Praktiken äußerlich, aber auch nicht die Letztursache (das wäre schlechter Idealismus); und die Begriffsarbeit ist keine reine Begriffsanalyse, sondern immer mit Gesellschaftsanalyse und Zeitdiagnose verbunden.
} 
notwendig ist. Diese Option darf selbst dann nicht einfach ausgeschlossen werden, wenn momentan keine gesellschaftlicher Akteure vorhanden sind, die diese Transformation realisieren könnten.

Drittens sollte ein solches Forschungsprogramm einen von Begründungsprogrammen oft nahegelegten Monismus vermeiden - denn nicht alle Phänomene lassen sich etwa durch das Prisma des kommunikativen Handelns oder der Anerkennung angemessen verstehen und kritisieren. ${ }^{14}$ Folter mag kommunikatives Handeln verunmöglichen und auch nicht auf reziprokes Verständnis abzielen; es mag eine Missachtung von Anerkennung beinhalten und die eigene Anerkennung erschweren; aber zu denken, Folter sei nur deshalb falsch und verurteilenswert, scheint mir etwas Wesentliches misszuverstehen. Folter ist auch deshalb falsch, weil zu ihr gravierende, unnötige Schmerzen gehören. Das allein ist schon Grund genug, sie zu verurteilen. Und überhaupt ist es wohl am angemessensten Folter als Paradigma des Schlechten zu verstehen und anderes dadurch zu verurteilen, dass es Elemente mit Folter teilt.

Viertens sollte Pluralismus auch auf einer zweiten Achse, d.h. bezüglich der Konzeptualisierung der sozialen Pathologie, an den Tag gelegt werden manche Phänomene sind besser dadurch zu beschreiben, dass die Gesellschaft oder eines ihrer zentralen Teilsysteme „krank“ ist (wie z.B. die Institutionen der repräsentativen Demokratie, deren Disfunktionalität an Occupy als auch an Unruhen in Paris, London, und Fergusen abzulesen ist); andere Phänomene besser dadurch, dass die Gesellschaft Individuen krank macht (wie z.B. die ansteigende Rate an Depressionen seit den 70er Jahren oder die höhere

\footnotetext{
${ }^{14}$ Siehe auch, im Bezug auf Habermas, T. Stahl, „Habermas and the Project of Immanent Critique“, Constellations 20.4 (2013): 533-52.
} 
Selbstmordrate und Kindersterblichkeit in Ländern wo Austerität als Antwort auf die Finanzkrise 2008 gewählt wurde oder erzwungen worden ist).

Zuletzt sollten wir auch nicht vor ethischen Urteilen zurückschrecken der Rawls'sche Einwand, dass dies aus dem Pluralismus der Weltanschauungen folge, trifft nämlich genauso auf moralische Werte zu (und auf die Frage, was überhaupt als ,moralisch' gelten kann), wie auf ethische; und wenn wir uns der Idee verschreiben wollten, dass normative Kriterien allgemein teilbar sein müssen, dann hat die Negativität physischer Schmerzen oder psychopathologischer Phänomene wie Depression oder die Tragik des frühen Todes von Kindern weit höhere Chancen, dies zu erreichen als die bestimmte Ideen von Freiheit und Gleichheit, die von Rawls und anderen propagiert werden.

Ich habe hier nur sehr knapp Elemente eines Forschungsprogramms umrissen. Was sie in einer Konstellation zusammenhält und vereint, ist kein deduktives System, kein Begründungsprogramm und kein normativer Monismus, sondern das Interesse an der Aufhebung gesellschaftlichen Unrechts und Elends.

Was also ist orthodoxe Kritische Theorie? Es ist das Festhalten an diesem Interesse als einziger Instanz der kritischen Theorie; es ist das durch dieses Interesse geleitete Unterfangen durch Begriffsarbeit, Selbstreflexion, und kritische Bezugnahme auf die Sozial- und Humanwissenschaften zum Kampf gegen Elend, Unrecht und Unfreiheit beizutragen. ${ }^{15}$

\footnotetext{
${ }^{15}$ vgl. „Materialismus und Moral“, 131: „... die theoretische Seite der Anstrengungen, das vorhandene Elend abzuschaffen“.
} 